\title{
Multiplicity of singularities is not a bi-Lipschitz invariant
}

Lev Birbrair ${ }^{1}$, Alexandre Fernandes ${ }^{2}$, J. Edson Sampaio, Misha Verbitsky ${ }^{3}$

\begin{abstract}
It was conjectured that multiplicity of a singularity is bi-Lipschitz invariant. We disprove this conjecture, constructing examples of bi-Lipschitz equivalent complex algebraic singularities with different values of multiplicity.
\end{abstract}

\section{Contents}

1 Introduction $\quad 1$

2 Smale-Barden manifolds 2

3 Multiplicity of homogeneous singularities 4

4 Lipschitz invariance of singularities 5

\section{Introduction}

The famous multiplicity conjecture, stated by Zariski in 1971 (see [Z2]), is formulated as follows: if two germs of complex analytic hypersurfaces are ambient topolological equivalent, then they have the same multiplicity. It was proved by Zariski [Z1] for germs of plane analytic curves. The results of Pham-Teisser in $[\mathrm{PT}]$ show that this result can be extended in the following "metric" way: if the two germs of complex analytic curves in n-dimensional space are bi-Lipschitz equivalent with respect to the outer metric, then the germs of the space curves have the same multiplicity. Comte in [Co] proves that the multiplicity of complex analytic germs (not necessarily codimension 1 sets) is invariant under bi-Lipschitz homeomorphism with Lipschitz constants close enough to 1 (this is a severe assumption). These results motivated the following question, closely related to the multiplicity conjecture:

\footnotetext{
${ }^{1}$ Partially supported by CNPq grant 302655/2014-0.

${ }^{2}$ Partially supported by CNPq grant 302764/2014-7.

${ }^{3}$ Partially supported by the Russian Academic Excellence Project '5-100'.
} 
is the multiplicity of a germ of analytic singularity a bi-Lipschitz invariant? This question was stated as a conjecture in [BFS].

The Lipschitz Regularity Theorem in [BFLS] shows that if the multiplicity of a complex analytic germ is equal to one, then it is a bi-Lipschitz invariant. Namely, if a germs of an analytic set is bi-Lipschitz equivalent to a smooth germ, then it is smooth itself. Later, Fernandes and Sampaio in [FS] give a positive answer to this question for surfaces in 3-dimensional space with respect to the ambient bi-Lipschitz equivalence. More recently, Neumann and Pichon ([NP]) showed that the multiplicity is an invariant under bi-Lipschitz equivalence, for germs of normal surface singularities. Another important result in $[\mathrm{FS}]$ is the following: in order to prove (or disprove) the bi-Lipschitz invariance of the multiplicity, it is enough to prove it for the algebraic cones, i.e. for the algebraic sets, defined by homogeneous polynomials. In [BFS] the authors show that the conjecture has a positive answer for 1 or 2 dimensional complex analytic sets.

The present paper shows that the multiplicity of complex algebraic sets is not a bi-Lipschitz invariant for the sets of dimension bigger or equal to three. Moreover, we show that there exists an infinite family of 3-dimensional germs, such that all of them are bi-Lipschitz equivalent, but they have different multiplicities. The idea of the construction is to consider the complex cones over different embeddings of $\mathbb{C} P^{1} \times \mathbb{C} P^{1}$ to complex projective spaces. Using the topology of Smale-Barden manifolds, we show that all the links of such singularities are diffeomorphic. That is why the germs of the corresponding cones are bi-Lipschitz equivalent. From the other hand, the multiplicities of the cones at the origin may be explicitly calculated in terms of the embeddings.

\section{Smale-Barden manifolds}

The classification of 5-manifolds is due to S. Smale ([S]) and D. Barden ([B]).

Definition 2.1: A simply connected, compact, oriented 5-manifold is called Smale-Barden manifold.

The Smale-Barden manifolds are uniquely determined by their second Stiefel-Whitney class and the linking form. 
Theorem 2.2: ([B] $)$ Let $X, X^{\prime}$ be two Smale-Barden manifolds. Assume that $H^{2}(X)=H^{2}\left(X^{\prime}\right)$ and this isomorphism is compatible with the linking form and preserves the second Stiefel-Whitney class. Then $X$ is diffeomorphic to $X^{\prime}$

Corollary 2.3: There exists only two Smale-Barden manifolds $M$ with $H^{2}(M)=\mathbb{Z}$, the product $S^{2} \times S^{3}$ and the total space of a non-trivial $S^{3}$ bundle over $S^{2}$ (see [C] for an introduction to Barden theory, where this manifold is formally introduced).

Proof: Indeed, the linking form on $\mathbb{Z}$ vanishes, therefore the manifold is uniquely determined by the Stiefel-Whitney class $w_{2}(M)$. Hence we have only two possibilities: $w_{2}(M)=0$ and $w_{2}(M) \neq 0$.

In early 2000-ies, the classification of 5-manifolds attracted interest coming from algebraic geometry, in the context of Sasakian geometry and geometry of generalized Seifert manifolds ([K1], [K2]). In the present paper we are interested in $S^{1}$-bundles over $\mathbb{C} P^{1} \times \mathbb{C} P^{1}$.

Proposition 2.4: Let $\pi: M \longrightarrow B$ be a simply connected 5-manifold obtained as a total space of an $S^{1}$-bundle $L$ over $B=\mathbb{C} P^{1} \times \mathbb{C} P^{1}$. Then $H^{2}(M)$ is torsion-free, and $M$ is diffeomorphic to $S^{2} \times S^{3}$.

Proof. Step 1: Universal coefficients formula gives an exact sequence

$$
0 \rightarrow \operatorname{Ext}_{\mathbb{Z}}^{1}\left(H_{1}(M ; \mathbb{Z}), \mathbb{Z}\right) \rightarrow H^{2}(M ; \mathbb{Z}) \rightarrow \operatorname{Hom}_{\mathbb{Z}}\left(H_{2}(M ; \mathbb{Z}), \mathbb{Z}\right) \rightarrow 0 .
$$

This implies that $H^{2}(M ; \mathbb{Z})$ is torsion-free.

Step 2: Consider the following exact sequence of homotopy groups

$$
0 \longrightarrow \pi_{2}(M) \longrightarrow \pi_{2}(B) \stackrel{\phi}{\longrightarrow} \pi_{1}\left(S^{1}\right) \longrightarrow \pi_{1}(M) \longrightarrow 0
$$

Since $\pi_{1}(M)=0$, the map $\phi$, representing the first Chern class of $L$, is surjective. This exact sequence becomes

$$
0 \longrightarrow \pi_{2}(M) \longrightarrow \mathbb{Z}^{2} \longrightarrow \mathbb{Z} \longrightarrow 0
$$

giving $\pi_{2}(M)=\mathbb{Z}$, and $H^{2}(M)=\mathbb{Z}$ because $H^{2}(M)$ is torsion-free. 
Step 3: To deduce Proposition 2.4 from the Smale-Barden classification, it remains to show that $w_{2}(M)=0$. However, $w_{2}(M)=\pi^{*}\left(\omega_{2}(B)\right)([\mathrm{K} 2$, Lemma 36]), and the latter clearly vanishes, because $w_{2}\left(S^{2}\right)=0$.

\section{Multiplicity of homogeneous singularities}

Given a projective variety $X \subset \mathbb{C} P^{n}$, the projective cone of $X$ is the union of all 1-dimensional subspaces $l \subset \mathbb{C}^{n+1}$ such that $l$, interpreted as a point in $\mathbb{C} P^{n}$, belongs to $X$. The link of $C(X)$ is an intersection of $C(X)$ with a unit sphere $S^{2 n+1} \subset \mathbb{C}^{n+1}$.

Let $A$ be complex algebraic set of $\mathbb{C}^{n+1}$ and $x \in A$. The multiplicity of $A$ at $x$, denoted by $\operatorname{mult}(A, x)$, is defined to be the multiplicity of the maximal ideal of the local ring $\mathcal{O}_{A, x}$. Given a projective variety $X \subset \mathbb{C} P^{n}$, we see that multiplicity of the projective cone $C(X)$ at the origin $0 \in \mathbb{C}^{n+1}$ coincides with degree of $X$ (see [Ch], subsection 11.3).

Next, we shall be interested in the following geometric situation. Let $X \subset \mathbb{C} P^{n}$ be a variety isomorphic to $\mathbb{C} P^{1} \times \mathbb{C} P^{1}$. Then the Picard group of $X=\mathbb{C} P^{1} \times \mathbb{C} P^{1}$ is isomorphic to $\mathbb{Z}^{2}$, and a line bundle is determined by its bidegree. We shall denote a line bundle of bidegree $a, b$ by $\mathcal{O}(a, b)$.

Proposition 3.1: Let $X \subset \mathbb{C} P^{n}$ be a variety isomorphic to $\mathbb{C} P^{1} \times \mathbb{C} P^{1}$, and $S \subset C(X)$ be its link. Assume that and $\left.\mathcal{O}(1)\right|_{X}=\mathcal{O}(a, b)$. Then $X$ has degree $2 a b$. If, in addition, $a$ and $b$ are relatively prime, the link of $C(X)$ is diffeomorphic to $S^{2} \times S^{3}$.

Proof. Step 1: Since $c_{1}(\mathcal{O}(a, b))^{2}=2 a b$, and degree of a subvariety $X \subset \mathbb{C} P^{n}$ is its intersection with the top power of $\left.\mathcal{O}(1)\right|_{X}$, one has $\operatorname{deg} X=$ $2 a b$.

Step 2: Consider the homotopy exact sequence

$$
0 \longrightarrow \pi_{2}(S) \longrightarrow \pi_{2}(X) \stackrel{\phi}{\longrightarrow} \pi_{1}\left(S^{1}\right) \longrightarrow \pi_{1}(S) \longrightarrow \pi_{1}(X) \longrightarrow 0
$$

for the circle bundle $\pi: S \longrightarrow X$. Since the map $\phi$ represents the first Chern class of $\left.\mathcal{O}(1)\right|_{X}$, it is obtained as a quotient of $\mathbb{Z}^{2}$ by a subgroup generated by $(a, b)$, and this map is surjective because $a$ and $b$ are relatively prime. Then 
$\pi_{1}(S)=\pi_{1}(X)=0$, and Proposition 2.4 implies that $S$ is diffeomorphic to $S^{2} \times S^{3}$.

\section{Lipschitz invariance of singularities}

Let $X \subset \mathbb{C}^{n}$ be a complex variety. The induced metric from the Euclidean distance on $\mathbb{C}^{n}$ gives a distance on $X$; it is called the outer metric on $X$.

Definition 4.1: Let $X \subset \mathbb{C}^{n}$ and $X^{\prime} \subset \mathbb{C}^{n^{\prime}}$ be complex varieties equipped with the outer metrics, $x \in X, x^{\prime} \in X^{\prime}$ marked points. We say that $(X, x)$ is bi-Lipschitz equivalent to $\left(X^{\prime}, x^{\prime}\right)$ if there exist a neighborhoods $U$ of $x$ in $\mathbb{C}^{n}$ and $U^{\prime}$ of $x$ in $\mathbb{C}^{n}$, and a bi-Lipschitz homeomorphism of $X \cap U$ to $X^{\prime} \cap U^{\prime}$ mapping $x$ to $x^{\prime}$.

Definition 4.2: Let $X, X^{\prime} \subset \mathbb{C}^{n}$ be complex varieties equipped with the outer metrics, $x \in X, x^{\prime} \in X^{\prime}$ marked points. We say that $(X, x)$ is ambient bi-Lipschitz equivalent to $\left(X^{\prime}, x^{\prime}\right)$ if there exists a bi-Lipschitz equivalence of a neighbourhood $U$ of $x$ in $\mathbb{C}^{n}$ and a neighbourhood $U^{\prime}$ of $x$ in $\mathbb{C}^{n}$ mapping $X \cap U$ to $X^{\prime} \cap U^{\prime}$ and $x$ to $x^{\prime}$.

Actually, the two definitions above do not coincide. The ambient biLipschitz equivalence implies bi-Lipschitz equivalence, but the examples presented in $[\mathrm{BG}]$ show that the converse does not hold true in general.

As it was already mentioned in Introduction, it was conjectured in [BFS] that the multiplicity is a bi-Lipschitz invariant. We prove that this is false. Here is the main result of this paper.

Theorem 4.3: For each $n \geq 3$, there exists a family $\left\{Y_{i}\right\}_{i \in \mathbb{Z}}$ of $n$-dimensional complex algebraic varieties $Y_{i} \subset \mathbb{C}^{n_{i}+1}$ such that:

(a) for each pair $i \neq j$, the germs at the origin of $Y_{i} \subset \mathbb{C}^{n_{i}+1}$ and $Y_{j} \subset$ $\mathbb{C}^{n_{j}+1}$ are bi-Lipschitz equivalent, but $\left(Y_{i}, 0\right)$ and $\left(Y_{j}, 0\right)$ have different multiplicity.

(b) for each pair $i \neq j$, there are $n$-dimensional complex algebraic varieties $Z_{i j}, \widetilde{Z}_{i j} \subset \mathbb{C}^{n_{i}+n_{j}+2}$ such that $\left(Z_{i j}, 0\right)$ and $\left(\widetilde{Z}_{i j}, 0\right)$ are ambient biLipschitz equivalent, but $\operatorname{mult}\left(Z_{i j}, 0\right)=\operatorname{mult}\left(Y_{i}, 0\right)$ and $\operatorname{mult}\left(\widetilde{Z}_{i j}, 0\right)=$ $\operatorname{mult}\left(Y_{j}, 0\right)$ and, in particular, they have different multiplicity. 
Proof. Let $\left\{p_{i}\right\}_{i \in \mathbb{Z}}$ be the family of odd prime numbers. For each $i \in \mathbb{Z}$, let $L_{i}$ be a very ample bundle on $X=\mathbb{C} P^{1} \times \mathbb{C} P^{1}$ of bidegree $\left(2, p_{i}\right)$. Let $X_{i}$ be projective variety obtained by the embedding of the very ample bundle $L_{i}$. Consider the link of the singularity $S_{i}:=C\left(X_{i}\right) \cap S^{2 m_{i}+1}$, where $S^{2 m_{i}+1}$ is the unit sphere centered in $0 \in \mathbb{C}^{m_{i}+1}$. Then, for each pair $i \neq j$ the links $S_{i}, S_{j}$ are diffeomorphic to $S^{2} \times S^{3}$ (Proposition 3.1). In particular, $S_{i}$ to $S_{j}$ are bi-Lipschitz homeomorphic. Since a bi-Lipschitz map from $S_{i}$ to $S_{j}$ induces a bi-Lipschitz map of their cones, then the affine cones $\left(C\left(X_{i}\right), 0\right)$ and $\left(C\left(X_{j}\right), 0\right)$ are bi-Lipschitz equivalent, but $\operatorname{mult}\left(C\left(X_{i}\right), 0\right)=4 p_{i}$ and $\operatorname{mult}\left(C\left(X_{j}\right), 0\right)=4 p_{j}$ (Proposition 3.1). Thus, if for each $i \in \mathbb{Z}$ we define $Y_{i}:=C\left(X_{i}\right) \times \mathbb{C}^{n-3}$, then we have that the family $\left\{Y_{i}\right\}_{i \in \mathbb{Z}}$ satisfies the item (a), since $\operatorname{mult}\left(Y_{i}, 0\right)=\operatorname{mult}\left(C\left(X_{i}\right), 0\right)=4 p_{i}$, for all $i \in \mathbb{Z}$.

Concerning to the item (b), let $\phi_{i j}: Y_{i} \rightarrow Y_{j}$ be a bi-Lipschitz homeomorphism such that $\phi_{i j}(0)=0$. Let $\widetilde{\phi_{i j}}: \mathbb{C}^{n_{i}+1} \rightarrow \mathbb{C}^{n_{j}+1}\left(\right.$ resp. $\widetilde{\psi_{i j}}: \mathbb{C}^{n_{j}+1} \rightarrow$ $\mathbb{C}^{n_{i}+1}$ ) be a Lipschitz extension of $\phi_{i j}$ (resp. $\left.\psi_{i j}=\phi_{i j}^{-1}\right)$ (see $[\mathrm{Ki}],[\mathrm{M}]$ and $[\mathrm{W}])$. Let us define $\varphi, \psi: \mathbb{C}^{n_{i}+1} \times \mathbb{C}^{n_{j}+1} \rightarrow \mathbb{C}^{n_{i}+1} \times \mathbb{C}^{n_{j}+1}$ as follows:

$$
\varphi(x, y)=\left(x-\widetilde{\psi_{i j}}\left(y+\widetilde{\phi_{i j}}(x)\right), y+\widetilde{\phi_{i j}}(x)\right)
$$

and

$$
\psi(z, w)=\left(z+\widetilde{\psi_{i j}}(w), w-\widetilde{\phi_{i j}}\left(z+\widetilde{\psi_{i j}}(w)\right)\right) .
$$

It easy to verify that $\psi=\varphi^{-1}$ and since $\varphi$ and $\psi$ are composition of Lipschitz maps, they are also Lipschitz maps. Moreover, if $Z_{i j}=Y_{i} \times\{0\}$ and $\widetilde{Z}_{i j}=\{0\} \times Y_{j}$, we obtain that $\varphi\left(Z_{i j}\right)=\widetilde{Z}_{i j}$ (see $\left.[\mathrm{Sa}]\right)$. Therefore, $\left(Z_{i j}, 0\right)$ and $\left(\widetilde{Z}_{i j}, 0\right)$ are bi-Lipschitz equivalent, but $\operatorname{mult}\left(Z_{i j}, 0\right)=\operatorname{mult}\left(Y_{i}, 0\right)$ and $\operatorname{mult}\left(\widetilde{Z}_{i j}, 0\right)=\operatorname{mult}\left(Y_{j}, 0\right)$ and, in particular, they have different multiplicity.

\section{References}

[BFLS] L. Birbrair, A. Fernandes, Lê D. T. and J. E. Sampaio. Lipschitz regular complex algebraic sets are smooth. Proceedings of the American Mathematical Society 144 (2016), no. 3, 983-987. 
[BG] Lev Birbrair and Andrei Gabrielov. Ambient Lipschitz equivalence of real surface singularities. arXiv:1707.04951v2 [math.AG], (2017), to appear in IMRN.

[B] D. Barden, Simply connected five-manifolds. Ann. of Math. 82 (1965), no. $2,365-385$.

[BFS] J. de Bobodilla, A. Fernandes and E. Sampaio. Multiplicity and degree as bi-Lipschitz invariants for complex sets. arXiv:1706.06614v2 [math.AG], (2017).

[Ch] E. M. Chirka. Complex analytic sets Kluwer Academic Publishers, 1989.

[C] Diarmuid Crowley. 5-manifolds: 1-connected. Bulletin of the Manifold Atlas (2011), 49-55.

[Co] Georges Comte. Multiplicity of complex analytic sets and bi-Lipschitz maps. Real analytic and algebraic singularities (Nagoya/Sapporo/Hachioji, 1996) Pitman Res. Notes Math. Ser., 381 (1998), 182-188.

[F] A. Fernandes Topological equivalence of complex curves and bi-Lipschitz maps. Michigan Math. J. 51 (2003), 593-606.

[FS] A. Fernandes and J. Edson Sampaio. Multiplicity of analytic hypersurface singularities under bi-Lipschitz homeomorphisms. Journal of Topology 9 (2016), 927-933, .

[Ki] M. Kirszbraun. $\ddot{U}$ ber die zusammenziehende und Lipschitzsche Transformationen. Fundamenta Math. 22 (1934), no. 1, 77-108.

[K1] J. Kollár. Seifert Gm-bundles. arXiv:math/0404386v2 [math.AG], (2004).

[K2] J. Kollár. Circle actions on simply connected 5-manifolds, Topology 45 (2006), no. 3, 643-671.

[M] E. J. Mcshane. Extension of range of functions. Bull. Amer. Math. Soc. 40 (1934), 837-842.

[NP] W. Neumann and A. Pichon Lipschitz geometry of complex surfaces: analytic invariants and equisingularity. arXiv:1211.4897v3 [math.AG], (2016).

[Sa] J. Edson Sampaio Bi-Lipschitz homeomorphic subanalytic sets have biLipschitz homeomorphic tangent cones. Selecta Math. (N.S.), 22 (2016), no. $2,553-559$. 
[S] S. Smale. On the structure of 5-manifolds, Ann. of Math. 75 (1962), no. 2, $38-46$.

[PT] F. Pham and B. Teissier. Fractions lipschitziennes d'une algébre analytique complexe et saturation de Zariski. Centre de Mathématiques de l'Ecole Polytechnique (Paris), June 1969.

[W] H. Whitney, Analytic extensions of functions defined in closed sets. Transactions of the Amer. Math. Soc. 36 (1934), 63-89.

[Z1] Zariski, O. On the topology of algebroid singularities. Amer. J. Math. 54 453-465, 1932.

[Z2] ZARISKI, O. Some open questions in the theory of singularities. Bull. of the Amer. Math. Soc. 77 (1971), no. 4, 481-491.

Misha Verbitsky

Instituto Nacional de Matemática Pura e Aplicada (IMPA)

Estrada Dona Castorina, 110

JARDIM BOTÂNICO, CEP 22460-320

Rio DE JANEIRo, RJ - BRASIL

ALSO:

Laboratory of Algebraic Geometry,

National Research University Higher School of Economics,

Department of Mathematics, 6 Usacheva street, Moscow, Russia.

Lev Bribrair, Alexandre Fernandes and J. Edson Sampaio

Departamento de Matemática, Universidade Federal do Ceará,

Rua Campus do Pici, s/n, Bloco 914

PICI, CEP 60440-900

Fortaleza-CE, Brazil 